\title{
F-box protein FBX022 mediates polyubiquitination and degradation of KLF4 to promote hepatocellular carcinoma progression
}

\author{
Xin Tian ${ }^{1}$, Shundong Dai ${ }^{2,3}$, Jing Sun ${ }^{4}$, Guojiang Jin' ${ }^{5}$, Shenyi Jiang ${ }^{6}$, Fandong Meng ${ }^{1}$, \\ Yan $\mathrm{Li}^{1}$, Di Wu ${ }^{1}$, Youhong Jiang ${ }^{1}$ \\ ${ }^{1}$ Molecular Oncology Laboratory of Cancer Research Institute, The First Affiliated Hospital of China Medical University, \\ Shenyang, 110001, China \\ ${ }^{2}$ Department of Pathology, The First Affiliated Hospital and College of Basic Medical Sciences of China Medical University, \\ Shenyang , 110001, China \\ ${ }^{3}$ Institute of Pathology and Pathophysiology, Shenyang, 110001, China \\ ${ }^{4}$ Department of Immunology and Biotherapy, Liaoning Cancer Hospital and Institute, Shenyang, 110042, China \\ ${ }^{5}$ Department of Laboratory Medicine, The First Affiliated Hospital of China Medical University, Shenyang, 110001, China \\ ${ }^{6}$ Department of Rheumatology, The First Affiliated Hospital of China Medical University, Shenyang, 110001, China \\ Correspondence to: \\ Youhong Jiang, e-mail: jiang_youhong@yeah.net
}

Keywords: Kruppel-like factor 4, FBXO22, hepatocellular carcinoma, ubiquitination

Received: March 21, $2015 \quad$ Accepted: May 14, $2015 \quad$ Published: May 28, 2015

\section{ABSTRACT}

Kruppel-like factor 4 (KLF4), a member of the KLF family of transcription factors, has been considered as a crucial tumor suppressor in hepatocellular carcinoma (HCC). Using affinity purifications and mass spectrometry, we identified FBX022, Cullin 1 and SKP1 as interacting proteins of KLF4. We demonstrate that F-box only protein 22 (FBX022) interacts with and thereby destabilizes KLF4 via polyubiquitination. As a result, FBXO22 could promote HCC cells proliferation both in vitro and in vivo. However, KLF4 deficiency largely blocked the proliferative roles of FBXO22. Importantly, FBXO22 expression was markedly increased in human HCC tissues, which was correlated with down-regulation of KLF4. Therefore, our results suggest that FBXO22 might be a major regulator of HCC development through direct degradation of KLF4.

\section{INTRODUCTION}

KLF4, a member of the krüppel-like factor (KLF) transcription factor family, is a potential tumor suppressor in several types of human malignancies [1-4]. For instance, KLF4 was significantly down-regulated in prostate cancer cell lines compared with nontumorigenic prostate cells [5]. RNA activation-mediated overexpression of KLF4 inhibited prostate cancer cell proliferation and altered the expression of several downstream cell-cycle-related genes [5]. Besides, ablation of KLF4 in gastric progenitor cells promoted transformation of the gastric mucosa and tumorigenesis in the antrum in mice [6]. Moreover, KLF4 protein expression was decreased or lost in hepatocellular carcinoma (HCC) tissues and, in particular, lymph node metastases when compared with that in normal liver [7].
Deficiency of KLF4 expression was significantly associated with poor survival, and also a prognostic marker in HCC patients [7]. Indeed, KLF4 could inhibit cell proliferation, invasion and epithelial to mesenchymal transition (EMT) through up-regulation of vitamin D receptor (VDR) and repression of $\beta$-catenin and SLUG, respectively [7-10]. However, the molecular mechanisms for the down-regulation of KLF4 in HCC tissues remain poorly understood.

It has been shown that KLF4 protein could be regulated by acetylation, phosphorylation and sumoylation [11-14]. More importantly, Lim KH et al. reports that KLF4 undergoes proteasomal degradation and some lysine residues are critical for its ubiquitination [15]. However, the factors regulating the ubiquitination and degradation of KLF4 have not been fully understood. 
The Skp1-Cul1-F box protein ubiquitin ligases (SCFs), consisting of Skp1, Cul1, and one of a member of $\mathrm{F}$ box proteins, play important roles in various biological events by their ability to bind and destabilize substrates $[16,17]$. For instance, FBXW7 could inhibit tumor growth by targeting Cyclin E, c-myc and SRC-3, all of which are thought to be oncoproteins [18-21]. Indeed, FBXW7 mutations have been found in a variety of primary human tumor types, providing novel insights into cancer progression and development of therapeutic drugs [22, 23]. In the current study, we used an immunoprecipitation and tandem mass spectrometry (IP-MS) analyses to search for KLF4-interacting protein with potential ubiquitin ligase activity and identified FBXO22 that binds with KLF4.

\section{RESULTS}

\section{Identifying FBXO22 as a KLF4-interacting protein}

To identify novel KLF4-interacting proteins, HepG2 cells were transfected with adenovirus expressing Flag-KLF4 or empty vector for $36 \mathrm{hr}$. Then proteins were isolated by Flag M2 beads, separated by SDS-PAGE,

A

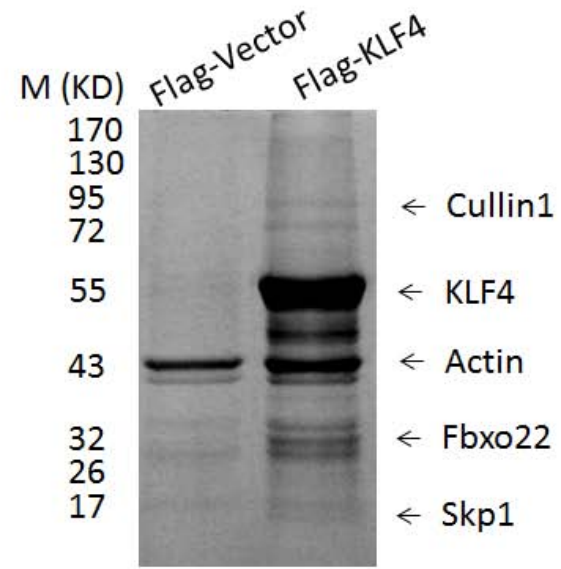

B

D

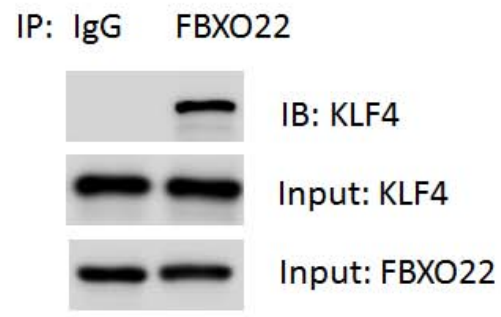

and identified by tandem mass spectrometry (MS/ MS). As shown in the Figure 1A, the examination of copurified endogenous proteins revealed the presence of unique peptides derived from SCF subunits, including Cullin1, SKP1 and F-box protein FBXO22 as major KLF4-associated proteins. FBXO22 is of interest since the F-Box protein family has been reported as the largest and most versatile class of E3 ubiquitin ligase. A FBXO22-KLF4 interaction was further confirmed by substantial portion of endogenous FBXO22 and KLF4 co-immunoprecipitated together (Figure $1 \mathrm{~B}$ and $1 \mathrm{C}$ ). This interaction was largely attenuated in KLF4knockdown cells, suggesting the specificity of this interaction (Figure 1D). To confirm the specific binding between KLF4 and FBXO22, we screened 11 human F box proteins. These FLAG tagged F-box proteins were transfected into $293 \mathrm{~T}$ cells and then immunoprecipitated to evaluate their interaction with endogenous KLF4. We found that, although all F-box proteins could interact with SKP1, only FBXO22 interacts with endogenous KLF4 (Supplementary Figure 1). In contrast, related $\mathrm{F}$ box proteins such as FBXO4, FBXO6, FBXO30, FBXO34, or FBXO36, which all belong to F-box only (FBXO) proteins, did not bind endogenous KLF4.

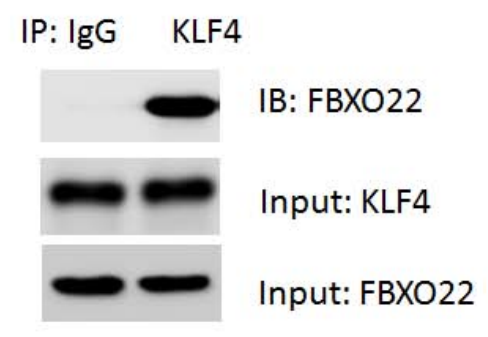

$\begin{array}{rlll}\text { shRNA: NC } & \text { KLF4 } & \text { NC } & \text { KLF4 } \\ \text { IP: IgG } & \text { IgG } & \text { FBXO22 } & \text { FBXO22 }\end{array}$

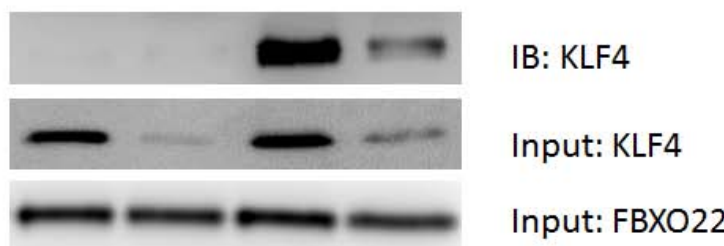

Figure 1: FBXO22 interacts with KLF4 in vivo. A. HepG2Cells were transfected with adenovirus expressing Flag-KLF4 or empty vector for $36 \mathrm{hr}$. The pull-down products from Flag M2 beads affinity purification were separated by SDS-polyacrylamide gel electrophoresis (SDS-PAGE), visualized by Colloidal blue staining, and analyzed by mass spectrometry. The identified SCF ${ }^{\mathrm{FBXO} 2}$ components are listed. B-C. Endogenous association between FBXO22 and KLF4 in HepG2 cells was performed by co-immunoprecipitation (CO-IP) experiments. D. The interaction of FBXO22 and KLF4 in cells depleted with KLF4. HepG2 cells were administered with adenoviral shRNA targeting KLF4 or a negative control (NC) 


\section{FBXO22 promotes KLF4 ubiquitination and degradation}

Members of F-Box proteins could recognize and mediate the degradation of target proteins. Thus, the fact that $\mathrm{FBXO} 22$ interacts with KLF4 prompted us to ask whether FBXO22 can promote the degradation of KLF4 as well. To test this hypothesis, adenovirus containing FBXO22 or GFP was introduced into HepG2 cells. Forced expression of FBXO22 led to a decreased protein levels of KLF4 (Figure 2A). The effect on KLF4 stability is selective since other members of KLF family (KLF2 and KLF5) were unaffected by FBOXO22 overexpression (Figure 2A). Besides, the reduction in KLF4 protein is not due to changes at the KLF4 mRNA levels, as shown by quantitative real-time PCR (Supplementary Figure 2). The decrease of KLF4 was also observed when FBXO22 was overexpressed in other HCC cells (HuH7 and Hep3B) and normal hepatocytes (L02) (Supplementary Figure 3A-3C). Ubiquitin-conjugation to KLF4 was enhanced by $\mathrm{FBXO} 22$ overexpression (Figure 2B). Besides, p2 $1^{\mathrm{Cip} / \mathrm{WAF} 1}$ and Cyclin B1, two cell-cycle regulators, were activated and repressed by KLF4 respectively [24, 25] and modulated by FBXO22 (Supplementary Figure 3D-3E).

To further analyze the FBXO22-mediated downregulation of KLF4 in more detail, degradation kinetics of KLF4 was determined in HepG2 cells. As expected, expression of FBXO22 significantly reduced the half-life of KLF4 (Figure 2C). On the other hand, we examined whether knockdown of FBXO22 could inhibit KLF4 turnover. FBXO22 deficiency resulted in an increased expression and half-life of KLF4 (Figure 2D-2E, Supplementary Figure 4A-4C), indicating that FBXO22 contributes to the turnover of KLF4.

\section{FBXO22 promotes HCC cell proliferation and invasion in vitro}

Given that KLF4 appears to function as a tumor suppressor in HCC, we next determined what physiologically stimulates $\mathrm{FBXO} 22$ to interact with and stabilize KLF4. As a result, the interaction of KLF4 and FBXO22 was enhanced during the cellcycle progression, leading to a reduced protein levels of KLF4 (Supplementary Figure 5). Besides, abilities of cell proliferation, colony formation and invasion were significantly enhanced by $\mathrm{FBXO} 22$ overexpression (Figure 3A-3C). In contrast, disruption of FBXO22 in HepG2 cells inhibited cell growth (Figure 3D-3F). Additionally, KLF4 deficiency largely blocked the proliferative roles of FBXO22 in HepG2 cells (Figure 4A-4B). In agreement, expression levels of
A

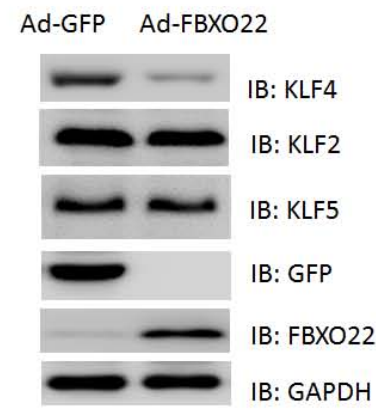

C

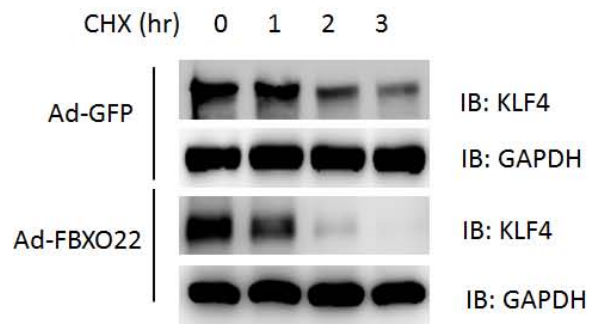

B

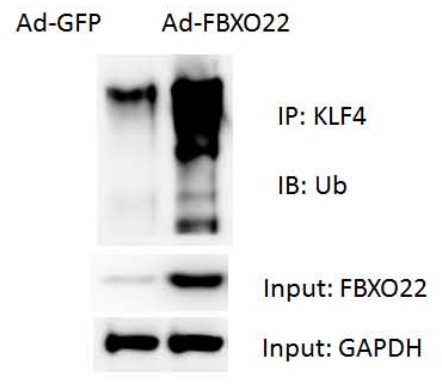

E $\mathrm{CHX}(\mathrm{hr}) \quad 0 \quad 1 \quad 2 \quad 3$
ShRNA-NC ShRNA-FBXO22

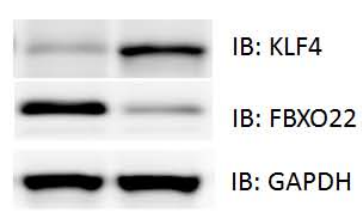

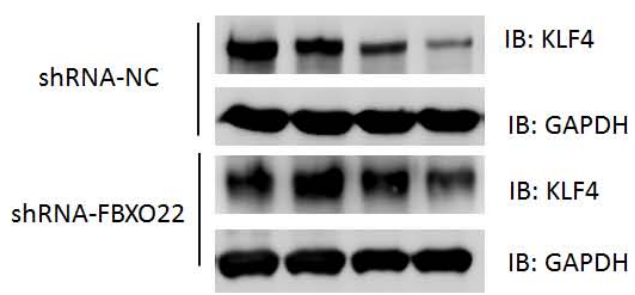

Figure 2: FBXO2 promotes KLF4 turnover in HCC cells. A. The levels of KLF4, KLF2 and KLF5 in cell lysates were determined by Western blot. HepG2 cells were transfected with adenovirus expressing GFP or FBXO22 for 36 hr. B. Ubiquitination of KLF4 in wholecell lysates was determined by Western blot. C. HepG2 cells were transfected with adenovirus expressing GFP or FBXO22. 36 hr after transfection, the cells were either lysed directly or incubated in the presence of cyclohexamide $(50 \mu \mathrm{g} / \mathrm{ml})$ for the indicated time period in order to determine KLF4 turnover. D. The levels of KLF4 in cell lysates were determined by Western blot. HepG2 cells were transfected with adenoviral shRNA targeting FBXO22 or a negative control (NC) for $36 \mathrm{hr}$. E. KLF4 turnover was determined by Western blot in HepG2 cells transfected with adenoviral shRNA targeting FBXO22 or a negative control (NC) for $36 \mathrm{hr}$. 
A

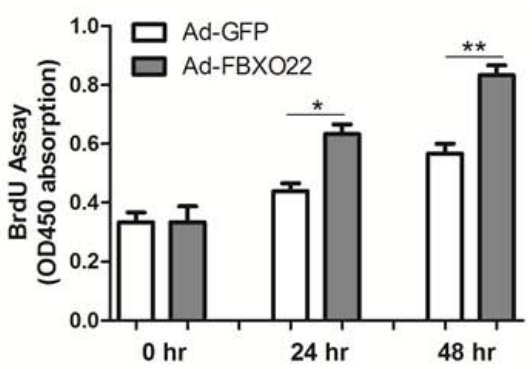

D

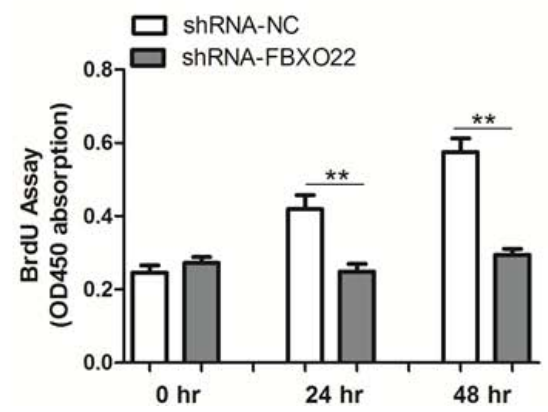

B

C

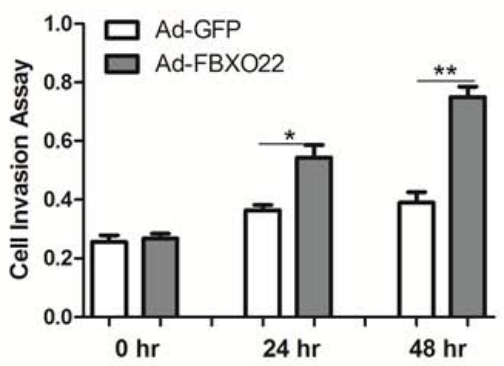

$\mathbf{E}$

$\mathbf{F}$

ShRNA-NC ShRNA-FBXO22
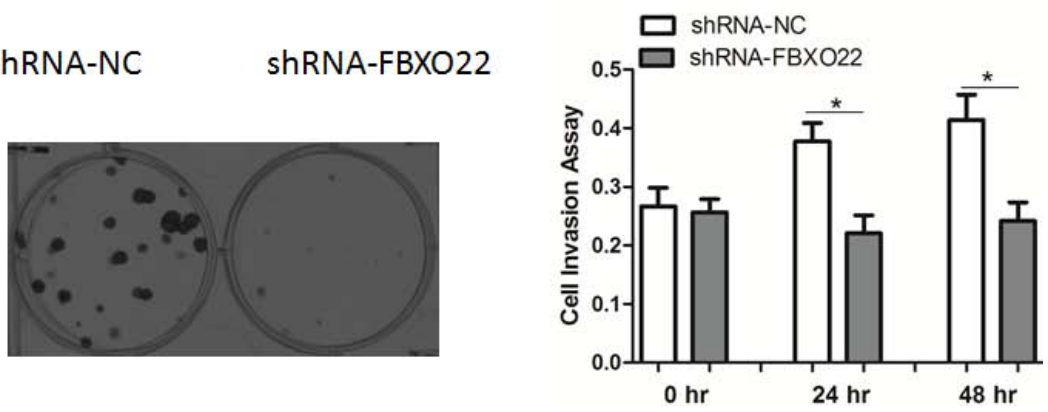

Figure 3: FBXO22 promotes HCC cell proliferation and invasion. Cell proliferation A, D., colony formation B, E. and invasion C, F. assays were determined in HepG2 cells with FBXO22 overexpression or knockdown.

A

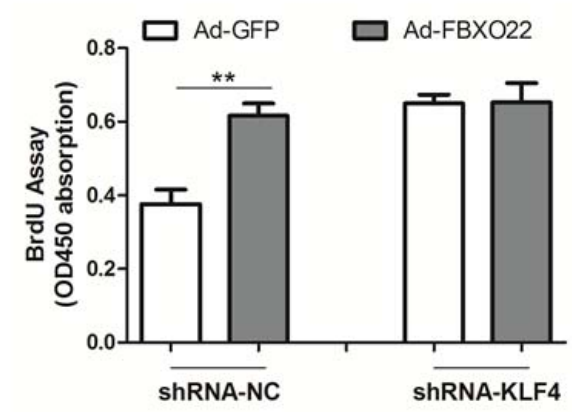

C

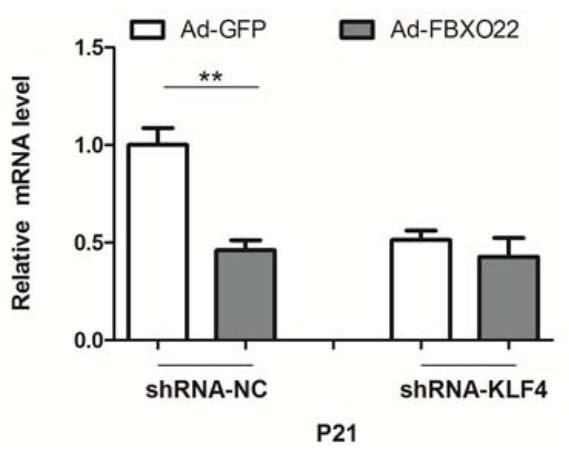

B
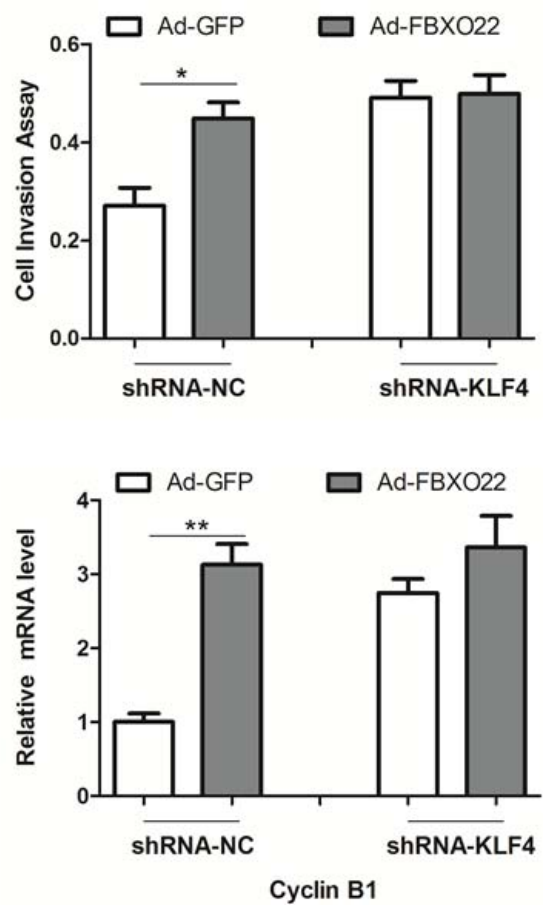

Figure 4: The oncogenic roles of FBXO22 rely on its regulation of KLF4. A-B. Cell proliferation (A) and invasion (B) assays in cells with FBXO22 overexpression and (or) KLF4 knockdown as indicated. HepG2 cells were transfected with adenovirus expressing GFP or FBXO22 for $24 \mathrm{hr}$, and then transfected with adenoviral shRNA targeting KLF4 or a negative control (NC) for another $24 \mathrm{hr}$. C. Relative mRNA levels of p21 and Cyclin B1 in HepG2 cells as in (A-B) 
p21 $1^{\mathrm{Cip} 1 / \mathrm{WAF} 1}$ and Cyclin B1 inhibited by FBXO22 were also attenuated by KLF4 silencing (Figure 4C), suggesting the oncogenic effects of FBXO22, at least in part, depend on its regulation of KLF4.

\section{FBXO22 promotes HCC growth in vivo}

To demonstrate the function of $\mathrm{FBXO} 22$ in vivo, HepG2 cells stably overexpressing FBXO22 or GFP protein were injected subcutaneously into two bilateral sites of $\mathrm{BALB} / \mathrm{c}$ nude mice. In agreement, the tumor volume and weight were markedly increased in FBXO22-overexpressed tumors compared to control tumors (Figure 5A-5B). KLF4 protein contents were reduced in $\mathrm{FBXO} 22$ overexpressed tumor tissues (Figure 5C), suggesting an oncogenic role of FBXO22 in vivo. Consistently, in FBXO22 overexpressed tumor tissues, the expression levels of p21 $1^{\text {Cip1/WAF1 }}$, and Cyclin B1 were also affected (Figure 5D-5E).

\section{Up-regulation of $\mathrm{FBXO22}$ in human hepatocarcinoma}

Finally, we asked whether FBXO22-mediated KLF4 degradation is involved in human HCC development. As shown in the Figure 6A, the expression of FBXO22 was significantly increased in tumor samples compared with adjacent normal tissue. In contrast, down-regulation of KLF4 was detected in tumor tissues (Figure 6A). Association analysis of $30 \mathrm{HCC}$ tissues indicated that the protein levels of FBXO22 and KLF4 was negatively correlated (Figure 6B), supporting the connection between FBXO22 and KLF4.

It has been well-established that inflammatory process is tightly associated with many different cancer types, including HCC. We speculate that the up-regulation of FBXO22 in HCC tissues might be attributed to aberrant activation of inflammatory signaling. Therefore, HepG2 and $\mathrm{HuH} 7$ cells were treated with TNF $\alpha$ and IL-1 $\beta$. As expected, treatment of these pro-inflammatory cytokines significantly increased the mRNA and protein levels of FBXO22 (Supplementary Figure 6A-6D). These results suggest that the up-regulation of FBXO22 in HCC tissues, at least in part, due to persistent activation of inflammatory signaling.

\section{DISCUSSION}

In the present study, we have demonstrated that KLF4 is a biological target of FBXO22, which promotes the turnover of KLF4 and thereby promotes HCC cell proliferation and invasion. It has been shown that promoter methylation of KLF4 locus could contribute to its down-regulation in vitro and in vivo [26, 27]. KLF4 was also identified as a direct target of several
A

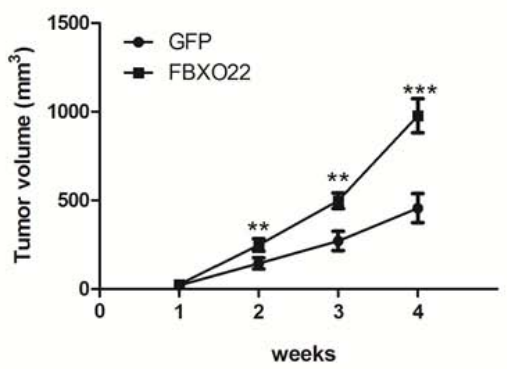

D

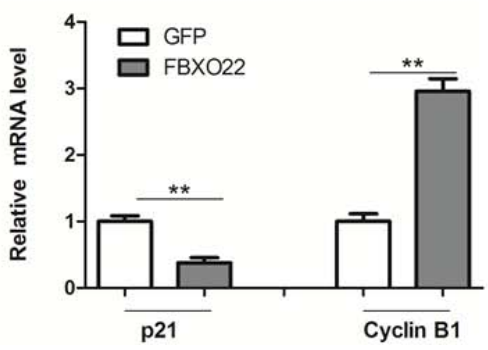

B

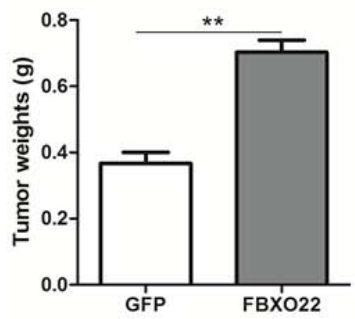

$\mathbf{E}$

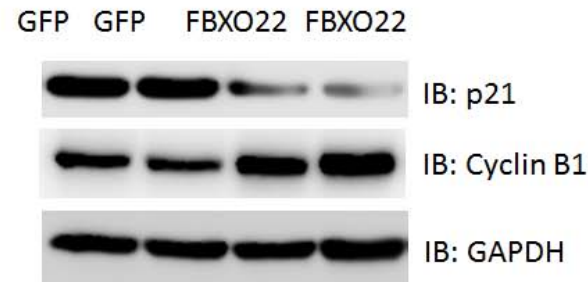

IB: FBXO22

IB: GFP

IB: KLF4

IB: GAPDH

Figure 5: FBXO22 promote HCC growth in vivo. A-B. HepG2 cells stably transfected with FBXO22 or GFP were injected into nude mice and followed up for tumorigenesis. Growth curve of tumor volumes (A) and tumor weights (B) were taken 4 weeks after injection. ( $n=6-8$ for each group) C. Representative protein levels of FBXO22, GFP and KLF4 were determined in the two groups of tumors. D-E. mRNA and protein levels of p21 and Cyclin B1 in two groups of tumors were determined by real-time PCR and Western blot. 
Patient \#1

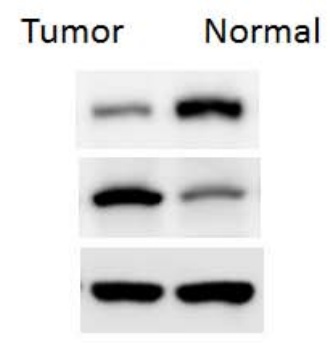

Patient \#3

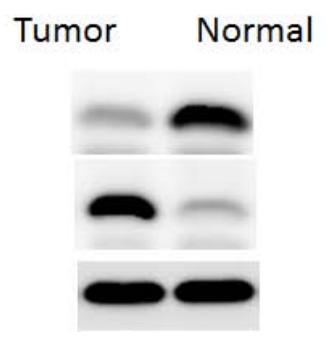

Patient \#2

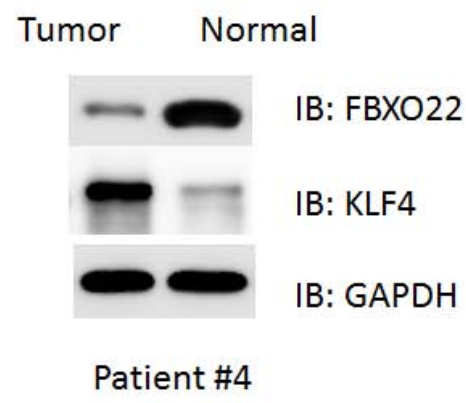

Tumor Normal

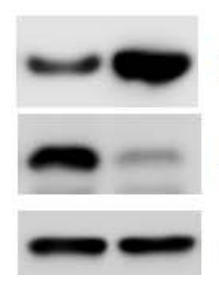

IB: FBXO22

IB: KLF4

IB: GAPDH

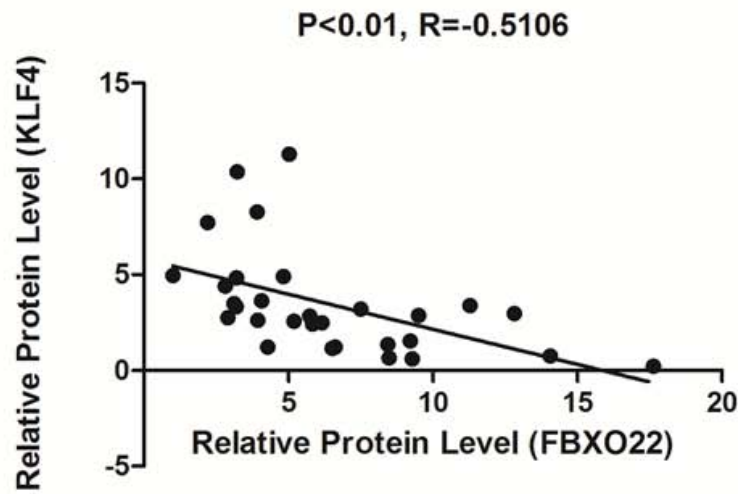

Figure 6: FBXO22 expression was increased in human hepatocarcinoma, correlating with reduced KLF4 expression. A. Protein levels of FBXO22 and KLF4 were determined by Western blot in human HCC tissues and adjacent normal tissues. B. The correlation between FBXO22 and KLF4 protein levels in paired HCC and non-tumor tissues.

microRNAs, a class of small and non-coding RNA molecules [28, 29]. Besides, TGF- $\beta$ signaling could promote KLF4 degradation through Cdh1-anaphase promoting complex [30]. In addition, Phosphorylation of KLF4 by ERK1 or ERK2 enhanced its interaction with the F-box proteins $\beta \operatorname{TrCP} 1$ or $\beta \operatorname{TrCP} 2$, which led to KLF4 ubiquitination and degradation [13]. Because KLF4 is a tumor suppressor clearly involved in the initiation and (or) progression of $\mathrm{HCC}$, it would be reasonable that important regulatory checks on its expression may exist at multiple levels. Therefore, our data present a novel mechanism for the dysregulation of KLF4 in HCC. However, further studies are still needed to establish the connection between FBXO22 and KLF4 in other gastrointestinal cancer.

Tan MK et al. reports that FBXO22 could control the activity of KDM4A through targeting it for proteasomal turnover [31], by which $\mathrm{FBXO} 22$ regulates histone $\mathrm{H} 3$ lysine 9 and 36 methylation levels in cell cycle. Besides, a recent study indicates that $\mathrm{FBXO} 22$ is essential for optimal synthesis of the N-methyl-D-aspartate (NMDA) receptor coagonist D-serine [32]. Here, we provide evidence that FBXO22 is up-regulated and might be an oncogene in HCC. However, the expression and roles of FBXO22 in other types of human cancers remain to be determined.

In conclusion, our study now suggests that FBXO22 controls the turnover of KLF4 protein in vivo and in vitro. Inhibition or down-regulation of $\mathrm{FBXO} 22$ by gene therapy might be beneficial in patients with malignant tumors.

\section{MATERIALS AND METHODS}

\section{Human tissue samples}

30 paired of HCC tissues and adjacent non-tumor normal tissues were collected from routine therapeutic surgery at our department. All samples were obtained with informed consent and approved by the hospital institutional review board.

\section{Cell culture}

HCC cell lines (HepG2, HuH7 and Hep3B cells) were obtained from The Cell Bank of Type Culture Collection of Chinese Academy of Sciences (CAS, Shanghai). Cells were grown in Dulbecco's modified Eagle's medium (DMEM, Gibco, Shanghai) supplemented with $10 \%$ fetal bovine serum (Gibco) and maintained at $37^{\circ} \mathrm{C}$ in a humidified atmosphere with $5 \% \mathrm{CO}_{2}$.

\section{Mouse experiments}

Male BALB/c nude mice aged 4 weeks were purchased from Shanghai Laboratory Animal Company (SLAC, Shanghai). $4.5 \times 10^{6}$ HepG2 cells stably expressing GFP or FBXO22 were injected subcutaneously to the skin under the front legs of the mouse. The mice were observed over 4 weeks for tumor formation. After the mice were sacrificed, the tumors were recovered and the wet weights of each tumor were determined. 


\section{Immunoprecipitation}

The purification process has been reported previously. Briefly, $5 \times 10^{7}$ cells infected with adenovirus expressing Flag-KLF4 or empty vector were lysed in $5 \mathrm{~mL}$ of NETN lysis buffer for $20 \mathrm{~min}$ on ice. Lysates were cleared using centrifugation at $13000 \mathrm{rpm}$ for $20 \mathrm{~min}$; the supernatant was then subjected to immunoprecipitation with $50 \mu \mathrm{L}$ of anti-FLAG M2 affinity resin overnight at $4^{\circ} \mathrm{C}$ with gentle inversion. Resin containing immune complexes was washed with $1 \mathrm{~mL}$ ice cold lysis buffer 6 times and proteins were eluted with $150 \mu \mathrm{L} 150 \mu \mathrm{g} / \mathrm{mL}$ $3 \times$ Flag-peptide (Sigma) in TBS for $10 \mathrm{~min}$. Proteins were precipitated with cold acetone.

\section{In gel tryptic digestion}

Immunoprecipitation samples were separated by SDS-PAGE, and visualized with colloidal Coomassie blue. The interest bands lane were cut into $1 \mathrm{~mm}$ slices, and each slice was washed twice with $50 \mathrm{mM} \mathrm{NH} 4 \mathrm{HCO} 3$, $50 \% \mathrm{ACN}$ and dehydrated with ACN. Proteins were reduced and alkylated by treating them with $10 \mathrm{mM}$ DTT and $55 \mathrm{mM}$ iodoacetamide, respectively. After washing with $50 \mathrm{mM}$ NH4HCO3 and $\mathrm{ACN}$, proteins were digested in gel with trypsin and incubated overnight at $37^{\circ} \mathrm{C}$. Tryptic peptides were extracted from the gel pieces with $60 \%$ ACN, $0.1 \%$ trifluoroacetic acid. The peptide extracts were vacuum centrifuged to dryness.

Samples were desalted with $10 \mathrm{ul}$ U-C18 pipette tips.

\section{Nano-HPLC-MS/MS Analysis}

The dried samples were dissolved in solvent A $(0.1 \%$ formic acid, $2 \%$ acetonitrile, $98 \% \mathrm{H} 2 \mathrm{O})$. Samples were then injected onto a manually packed reversed phase C18 column $(150 \mathrm{~mm} \times 79 \mu \mathrm{m}$, 3- $\mu \mathrm{m}$ particle size, Dikma, China) coupled to Easy nLC (Thermo Fisher Scientific, Waltham, MA). Peptides were eluted from $7 \%$ to $80 \%$ solvent B $\left(0.1 \%\right.$ formic acid in $90 \%$ acetonitrile and $\left.10 \% \mathrm{H}_{2} \mathrm{O}\right)$ in solvent $\mathrm{A}(0.1 \%$ formic acid in $2 \%$ acetonitrile and $98 \% \mathrm{H}_{2} \mathrm{O}$ ) with a $1 \mathrm{~h}$ gradient at a flow rate of $300 \mathrm{nl} / \mathrm{min}$. The fractions were analyzed by using a Q Exactive mass spectrometer in a top 16 data-dependent mode. For full MS spectra, the scan range was 350 to 1300 with a resolution of 70,000 . For MS/MS scan, the 16 most intense ions with charge state 2 and 3 in each full MS spectrum were sequentially fragmentated by higher energy collisional dissociation (HCD) with normalized collision energy of $28 \%$. The resolution was 17,500 . The dynamic exclusion duration was set to be $60 \mathrm{~s}$, and the isolation window was $1.5 \mathrm{~m} / \mathrm{z}$. All MS raw files were analyzed by MaxQuant software (version 1.0.13.13) and Mascot software (version 2.1) against the database uniprot_Human to identify proteins.

\section{Real-time PCR analysis}

Total RNA from tissues and cells was extracted using the RNA Isolation Kit (Takara, Dalian, China) according to the manufacturer's instructions. Quantitative real-time PCR was performed by using an Applied Biosystems 7300 Real-time PCR System and a TaqMan Universal PCR Master Mix. Expression of the KLF4, p21 and Cyclin B1 was normalized to that of the $\beta$-actin.

\section{Western blot}

Cells were harvested and lysed with ice-cold lysis buffer $(50 \mathrm{mM}$ Tris-HCl, $\mathrm{pH}$ 7.4, $100 \mathrm{mM}$ 2-Mercaptoethanol, $2 \% \mathrm{w} / \mathrm{v}$ SDS, $10 \%$ glycerol). After centrifugation at $10000 \times \mathrm{g}$ for $10 \mathrm{~min}$ at $4^{\circ} \mathrm{C}$, proteins in the supernatants were quantified and separated by $10 \%$ SDS PAGE. Western blot assay was performed using anti-FBXO22, KLF4, p21, Cyclin B1, GFP and GAPDH antibodies (Abcam, USA). GAPDH was determined as a loading control

\section{BrdU and cell invasion assays}

A cell proliferation enzyme-linked immunosorbent assay (BrdU kit; Beyotime) was used to analyze the incorporation of BrdU during DNA synthesis following the manufacturer's protocols. Absorbance was measured at $450 \mathrm{~nm}$ in the Spectra Max 190 ELISA reader (Molecular Devices, Sunnyvale, CA). For cell invasion assays, cells were analyzed using extracellular matrix-coated invasion chambers (Millipore, CA, USA), and quantitated with a colorimetric microplate reader at $570 \mathrm{~nm}$, according to the manufacturer's instructions.

\section{Colony formation assay}

Cells were seeded in a 6-well plate 48 hours posttransduction and cultured for 8 to 10 days at $37^{\circ} \mathrm{C}$ in $5 \% \mathrm{CO}_{2}$. Cells were fixed with $4 \%$ paraformaldehyde in phosphate-buffered saline (PBS), washed twice with PBS, and stained with a crystal violet solution ( $1 \%$ crystal violet, $10 \%$ ethanol in water). Stained cells were washed thrice with water and counted by under an optical microscope.

\section{Statistical analysis}

The data shown represent the mean \pm standard error (SE) values of three independent experiments. Significance was analyzed using Student's $t$-test $\left({ }^{*} p<0.05,{ }^{* *} p<0.01\right.$, $* * * p<0.001)$.

\section{ACKNOWLEDGMENTS}

This work was supported by grants from the National Natural Science Foundation of China (No. 81202955, 81401881 and 81372287). 


\section{CONFLICTS OF INTEREST}

The authors declared no conflict of interest.

\section{REFERENCES}

1. Cui J, Shi M, Quan M, Xie K. Regulation of EMT by KLF4 in gastrointestinal cancer. Current cancer drug targets. 2013; 13:986-995.

2. Evans PM, Liu C. Roles of Krupel-like factor 4 in normal homeostasis, cancer and stem cells. Acta biochimica et biophysica Sinica. 2008; 40:554-564.

3. Ghaleb AM, Nandan MO, Chanchevalap S, Dalton WB, Hisamuddin IM, Yang VW. Kruppel-like factors 4 and 5: the yin and yang regulators of cellular proliferation. Cell research. 2005; 15:92-96.

4. Wei D, Kanai M, Huang S, Xie K. Emerging role of KLF4 in human gastrointestinal cancer. Carcinogenesis. 2006; $27: 23-31$.

5. Wang J, Place RF, Huang V, Wang X, Noonan EJ, Magyar CE, Huang J, Li LC. Prognostic value and function of KLF4 in prostate cancer: RNAa and vector-mediated overexpression identify KLF4 as an inhibitor of tumor cell growth and migration. Cancer research. 2010; 70:10182-10191

6. Li Q, Jia Z, Wang L, Kong X, Li Q, Guo K, Tan D, Le X, Wei D, Huang S, Mishra L, Xie K. Disruption of Klf4 in villin-positive gastric progenitor cells promotes formation and progression of tumors of the antrum in mice. Gastroenterology. 2012; 142:531-542.

7. Li Q, Gao Y, Jia Z, Mishra L, Guo K, Li Z, Le X, Wei D, Huang S, Xie K. Dysregulated Kruppel-like factor 4 and vitamin $\mathrm{D}$ receptor signaling contribute to progression of hepatocellular carcinoma. Gastroenterology. 2012; 143:799-810. e791-792.

8. Thylur RP, Senthivinayagam S, Campbell EM, Rangasamy V, Thorenoor N, Sondarva G, Mehrotra S, Mishra P, Zook E, Le PT, Rana A, Rana B. Mixed lineage kinase 3 modulates beta-catenin signaling in cancer cells. The Journal of biological chemistry. 2011; 286:37470-37482.

9. Lin ZS, Chu HC, Yen YC, Lewis BC, Chen YW. Kruppellike factor 4, a tumor suppressor in hepatocellular carcinoma cells reverts epithelial mesenchymal transition by suppressing slug expression. PloS one. 2012; 7:e43593.

10. Lu XJ, Shi Y, Chen JL, Ma S. Kruppel-like factors in hepatocellular carcinoma. Tumour biology: the journal of the International Society for Oncodevelopmental Biology and Medicine. 2015; 36:533-541.

11. Evans PM, Zhang W, Chen X, Yang J, Bhakat KK, Liu C. Kruppel-like factor 4 is acetylated by p300 and regulates gene transcription via modulation of histone acetylation. The Journal of biological chemistry. 2007; 282:33994-34002.
12. Meng F, Han M, Zheng B, Wang C, Zhang R, Zhang XH, Wen JK. All-trans retinoic acid increases KLF4 acetylation by inducing HDAC2 phosphorylation and its dissociation from KLF4 in vascular smooth muscle cells. Biochemical and biophysical research communications. 2009; 387:13-18.

13. Kim MO, Kim SH, Cho YY, Nadas J, Jeong CH, Yao K, Kim DJ, Yu DH, Keum YS, Lee KY, Huang Z, Bode AM, Dong Z. ERK1 and ERK2 regulate embryonic stem cell self-renewal through phosphorylation of Klf4. Nature structural \& molecular biology. 2012; 19:283-290.

14. Tahmasebi S, Ghorbani M, Savage P, Yan K, Gocevski G, Xiao L, You L, Yang XJ. Sumoylation of Kruppel-like factor 4 inhibits pluripotency induction but promotes adipocyte differentiation. The Journal of biological chemistry. 2013; 288:12791-12804.

15. Lim KH, Kim SR, Ramakrishna S, Baek KH. Critical lysine residues of Klf4 required for protein stabilization and degradation. Biochemical and biophysical research communications. 2014; 443:1206-1210.

16. Skaar JR, Pagan JK, Pagano M. SCF ubiquitin ligasetargeted therapies. Nature reviews Drug discovery. 2014; 13:889-903.

17. Wang Z, Liu P, Inuzuka H, Wei W. Roles of F-box proteins in cancer. Nature reviews Cancer. 2014; 14:233-247.

18. Koepp DM, Schaefer LK, Ye X, Keyomarsi K, Chu C, Harper JW, Elledge SJ. Phosphorylation-dependent ubiquitination of cyclin E by the SCFFbw7 ubiquitin ligase. Science. New York, NY: 2001; 294:173-177.

19. Yada M, Hatakeyama S, Kamura T, Nishiyama M, Tsunematsu R, Imaki H, Ishida $\mathrm{N}$, Okumura $\mathrm{F}$, Nakayama K, Nakayama KI. Phosphorylation-dependent degradation of c-Myc is mediated by the F-box protein Fbw7. The EMBO journal. 2004; 23:2116-2125.

20. King B, Trimarchi T, Reavie L, Xu L, Mullenders J, Ntziachristos P, Aranda-Orgilles B, Perez-Garcia A, Shi J, Vakoc C, Sandy P, Shen SS, Ferrando A, Aifantis I. The ubiquitin ligase FBXW7 modulates leukemia-initiating cell activity by regulating MYC stability. Cell. 2013; 153:1552-1566.

21. Wu RC, Feng Q, Lonard DM, O'Malley BW. SRC-3 coactivator functional lifetime is regulated by a phospho-dependent ubiquitin time clock. Cell. 2007; 129:1125-1140.

22. Yumimoto K, Akiyoshi S, Ueo H, Sagara Y, Onoyama I, Ueo H, Ohno S, Mori M, Mimori K, Nakayama KI. F-box protein FBXW7 inhibits cancer metastasis in a non-cell-autonomous manner. The Journal of clinical investigation. 2015; 125:621-635.

23. Aydin IT, Melamed RD, Adams SJ, Castillo-Martin M, Demir A, Bryk D, Brunner G, Cordon-Cardo C, Osman I, Rabadan R, Celebi JT. FBXW7 mutations in melanoma and a new therapeutic paradigm. Journal of the National Cancer Institute. 2014; 106. 
24. Rowland BD, Peeper DS. KLF4, p21 and context-dependent opposing forces in cancer. Nature reviews Cancer. 2006; 6:11-23.

25. Yoon HS, Yang VW. Requirement of Kruppel-like factor 4 in preventing entry into mitosis following DNA damage. The Journal of biological chemistry. 2004; 279:5035-5041.

26. Jiang YZ, Jimenez JM, Ou K, McCormick ME, Zhang LD, Davies PF. Hemodynamic disturbed flow induces differential DNA methylation of endothelial Kruppel-Like Factor 4 promoter in vitro and in vivo. Circulation research. 2014; 115:32-43.

27. Nakahara Y, Northcott PA, Li M, Kongkham PN, Smith C, Yan H, Croul S, Ra YS, Eberhart C, Huang A, Bigner D, Grajkowska W, Van Meter T, Rutka JT, Taylor MD. Genetic and epigenetic inactivation of Kruppel-like factor 4 in medulloblastoma. Neoplasia. New York, NY: 2010; 12:20-27.

28. Ma J, Yao Y, Wang P, Liu Y, Zhao L, Li Z, Li Z, Xue Y. MiR-152 functions as a tumor suppressor in glioblastoma stem cells by targeting Kruppel-like factor 4. Cancer letters. 2014; 355:85-95.
29. Meza-Sosa KF, Perez-Garcia EI, Camacho-Concha N, Lopez-Gutierrez O, Pedraza-Alva G, Perez-Martinez L. MiR-7 promotes epithelial cell transformation by targeting the tumor suppressor KLF4. PloS one. 2014; 9:e103987.

30. Hu D, Wan Y. Regulation of Kruppel-like factor 4 by the anaphase promoting complex pathway is involved in TGFbeta signaling. The Journal of biological chemistry. 2011; 286:6890-6901.

31. Tan MK, Lim HJ, Harper JW. SCF(FBXO22) regulates histone $\mathrm{H} 3$ lysine 9 and 36 methylation levels by targeting histone demethylase KDM4A for ubiquitin-mediated proteasomal degradation. Molecular and cellular biology. 2011; 31:3687-3699.

32. Dikopoltsev E, Foltyn VN, Zehl M, Jensen ON, Mori H, Radzishevsky I, Wolosker H. FBXO22 protein is required for optimal synthesis of the N-methyl-D-aspartate (NMDA) receptor coagonist D-serine. The Journal of biological chemistry. 2014; 289:33904-33915. 